\title{
MEASURABLE, TAIL DISINTEGRATIONS OF THE HAAR INTEGRAL ARE PURELY FINITELY ADDITIVE ${ }^{1}$
}

\author{
LESTER E. DUBINS
}

\begin{abstract}
There are countably additive probability measures, $P$, and subsigma fields, relative to which $P$ admits no proper, measurable, conditional distributions, except, possibly, those which are purely finitely additive. The usual fair, coin-tossing probability measure and the tail sigma field illustrate this phenomenon. More generally, every measurable, disintegration of the Haar integral of any compact metrizable group, $G$, relative to the partition, $\Pi$, of $G$ which consists of the left cosets of any dense denumerable subgroup $S$ of $G$, or what comes to the same thing, relative to the sigma field of Haar-measurable subsets of $G$ which are invariant under right translation by $S$, is purely finitely additive.
\end{abstract}

This note relates to [1] and [2], but is logically independent of these references.

Let $S$ be a denumerable, dense subgroup of a compact, metrizable group, $G$, and let $M$ be the unique, $G$-invariant mean defined on the space $C(G)$ of all real-valued continuous functions on $G$. Let $\Pi=G / S$ be the set of left cosets $g S$ of $S$.

THEOREM 1. Every measurable, П-disintegration of $M$ is purely finitely additive.

To say that $\sigma$ is a measurable $\pi$-disintegration of $M$ means this. For all $f$ in the domain of $M$,

$$
M f=\int \sigma_{g}\left(f^{g}\right) d(g)
$$

where: (a) $f^{g}$ is the trace of $f$ on $g S$, that is, the restriction of $f$ to $g S$; (b) $\sigma_{g}$ is a mean which is supported by $g S$, so that $\sigma_{g}(g S)$ equals $1, \sigma_{g}=\sigma_{g^{\prime}}$ if $g S=g^{\prime} S$, and $\sigma_{g}$ is defined for $f^{g}$ for all $f$ in the domain of $M$, and (c) $\sigma_{g}\left(f^{g}\right)$ is integrable with respect to Haar measure $d g$.

If, except for a set of $g$ 's of Haar measure zero, $\sigma_{g}$ is purely finitely additive, $\sigma$ is said to be purely finitely additive.

The proof of Theorem 1 consists of two steps, the first of which is the

Received by the editors February 10, 1976.

AMS (MOS) subject classifications (1970). Primary 28A50, 60A05, $22 \mathrm{C} 05$.

Key words and phrases. Disintegrations, measures, conditional probability, compact groups, finite additivity, conglomerability.

1 This research was prepared with the support of the National Science Foundation, Grant No. MPS 75-09459. 
exhibition of a purely finitely additive disintegration $\hat{\sigma}$ of $M$, and the second is a demonstration that for every measurable disintegration $\sigma$ of $M, \hat{\sigma}_{g}=\sigma_{g}$ for almost all $g$.

Since, for each $g, g S$ is dense in $G$, the map $f \rightarrow f^{g}$ is a 1-1 mapping of $C(G)$ onto the space of uniformly continuous functions defined on $g S$. Consequently,

$$
\hat{\sigma}_{g}\left(f_{g}\right)=M f
$$

defines $\hat{\sigma}$ uniquely. It is straightforward to verify that $\hat{\sigma}$ is a measurable $\Pi$-disintegration of $M$, henceforth called elementary.

LEMMA 1. The elementary $\Pi$-disintegration of $M$ is purely finitely additive.

Proof. Let $\varepsilon>0$, and let $s_{1}, s_{2}, \ldots$ be an enumeration of the elements of $S$. As the Tietze extension theorem implies, there is, for each $n$, an $f_{n} \in$ $C(G), 0 \leqslant f_{n} \leqslant 1$, such that $f_{n}\left(s_{n}\right)$ is 0 and $f_{n}$ is less than 1 on an open set of Haar measure at most $\varepsilon 2^{-n}$. Let $f_{n}^{\prime}$ designate the infimum of $f_{i}, i=1, \ldots, n$, and verify: $f_{n}^{\prime} \in C(G) ; f_{n}^{\prime} \geqslant f_{n+1}^{\prime} ; f_{n}^{\prime}$ converges to 0 on $S$; and $M\left(f_{n}^{\prime}\right)$ exceeds $1-\varepsilon$. Consequently, the restriction to $S$ of the $f_{n}^{\prime}$ converges monotonely down to 0 everywhere on $S$ and, for any $g \in S, \hat{\sigma}_{g}\left(f_{n}^{\prime}\right)$ exceeds $1-\varepsilon$. This implies that, for $g \in S, \hat{\sigma}_{g}$ is purely finitely additive. By appropriately translating the sequence $f_{n}$, one concludes that every $\hat{\sigma}_{g}$ is purely finitely additive. Q.E.D.

A function $\phi$ defined on $G$ is $S$-invariant if, for every $s \in S$, the right translate of $\phi$ by $s$ is identical with $\phi$. If, for every integrable, $S$-invariant $\phi$, there is a constant $c$ such that $\phi=c$ almost certainly, then $S$ acts ergodically.

LEMMA 2. Every dense subset of $G$ acts ergodically.

Surely Lemma 2 is known, but since I know of no reference, I supply a proof.

Proof of Lemma 2. Let $\phi$ be integrable and $S$-invariant for the dense subset $S$ of $G$. Let $f \in C(G)$ approximate $\phi$ in the $L_{1}$ norm. As shown by von Neumann [3], [4], there is a finite sequence of elements of $G, g_{1}, \ldots, g_{n}$, such that the average, $F$, of the right translates $f_{i}$ of $f$ by the $g_{i}$ is uniformly close to a constant. Because $s_{i} \in S$ can be chosen arbitrarily close to $g_{i}$, it may be supposed that the $g_{i}$ themselves are in $S$. Of course, the corresponding right translates $\phi_{i}$ of $\phi$ approximate $f_{i}$ in the $L_{1}$ norm as well as $\phi$ approximates $f$. Consequently, the average, $\theta$, of the $\phi_{i}$ approximates $F$, the average of the $f_{i}$. But, since $\phi$ is $S$-invariant, $\theta$ is $\phi$. So $F$ approximates $\phi$ in $L_{1}$, which implies that, for every $\varepsilon>0$, there is a constant $c$ such that $|\phi-c| \leqslant \varepsilon$ except on a set of measure less than $\varepsilon$. This implies that a single constant $c$ satisfies this condition for all $\varepsilon>0$. Now invoke countable additivity to see that $\phi=c$ almost surely. Q.E.D.

Lemma 3. Let $\sigma$ be a measurable, П-disintegration of $M$. Then $\sigma_{g}=\hat{\sigma}_{g}$ for almost all $\mathrm{g}$. 
Proof. As implied by Lemma 2, for each $f \in C(G)$, there is a constant, $c(f)$, and a subset of $G$ of measure 0 , say $N(f)$, such that, for all $g$ in the complement of $N(f)$,

$$
\sigma_{g}\left(f^{g}\right)=c(f)
$$

In view of (1), $c(f)=M f$. Because $G$ is compact and metrizable, there is a countable subset $D$ of $C(G)$ which is dense in $C(G)$ for the topology of uniform convergence. Let $N^{c}$ be the complement of the union of the null sets $N(f)$ for $f$ in $D$. Summarizing, for all $g \in N^{c}$, for all $f \in D$,

$$
\sigma_{g}\left(f^{g}\right)=M f
$$

As is easily verified, for each $g$, the set of $f$ for which (4) holds is closed in the uniform topology. Consequently, for $g \in N^{c}$, (4) holds for all $f \in C(G)$. Equivalently, for all $g \in N^{c}, \sigma_{g}=\hat{\sigma}_{g}$. Q.E.D.

In view of Lemmas 1 and 3, Theorem 1 is seen to hold.

Consider now the special case in which $G$ is the product of a denumerable number of copies of the cyclic group of order two. The usual fair, coin-tossing, probability measure is identical with normalized Haar measure on $G$. And the atoms of the tail sigma field are simply the cosets of the subgroup $S$ consisting of those elements of $G$ which have no more than a finite number of nonzero coordinates. Hence, if Theorem 1 is applied to this example, one obtains

COROLlARY 1. Let $\Omega$ be the space of infinite sequences of zeroes and ones, $\mathscr{F}$ the field of finite-dimensional, Borel subsets of $\Omega, P$ the fair-coin probability measure on $\mathcal{F}$. Then every Lebesgue measurable, proper, conditional distribution of $P$ given the tail sigma field is purely finitely additive.

A similar example is obtained by letting $G$ be the unit interval, with addition taken mod $1, d g$ equal to Lebesgue measure, and $S \subset G$, the set of rationals.

Possibly the elementary $\Pi$-disintegration of $M$ can be extended so as to be a $\Pi$-disintegration of all integrable functions, but the contrary seems to me to be more likely. Indeed, it is not unlikely that every $\Pi$-disintegrable measure defined for all Borel subsets of $G$ is orthogonal to Haar measure on $G$. A related conjecture is that "measurable" can be deleted from the statement of Theorem 1.

\section{REFERENCES}

1. Lester E. Dubins and David Blackwell, On existence and non-existence of proper, regular, conditional distributions, Ann. Probability 3 (1975), 741-752.

2. Lester E. Dubins, Finitely additive conditional probabilities, conglomerability and disintegrations, Ann. Probability 3 (1975), 89-99. MR 50\# 11348.

3. John von Neumann, Zum Haarschen Mass in topologischen Gruppen, Compositio Math. 1 (1934), 106-114.

4. L. S. Pontryagin, Topological groups, 2nd ed., GITTL, Moscow, 1954; English transl., Gordon and Breach, New York, 1966. MR 17, 171; 34 \#1439.

Department of Mathematics, University of California, Berkeley, California 94720 